\title{
Production and Decay of HF in ATLAS
}

\author{
Angel Campoverde ${ }^{* \dagger}$ \\ University of Siegen \\ E-mail: angel.campoverde@cern.ch
}

\begin{abstract}
An angular analysis of the decay of $B_{d} \rightarrow K^{*} \mu^{+} \mu^{-}$is peformed for a number of angular coefficients, which are measured as a function of the invariant mass squared of the dimuon system for data collected at $\sqrt{s}=8 \mathrm{TeV}$ by ATLAS [2], at the LHC [1]. Comparisons are made to theoretical predictions, including for the observable $P_{5}^{\prime}$, for which there has been recent tension between theory and experiment. In addition a study of kinematical correlations in $B$ hadron pair production is presented.
\end{abstract}

XXVI International Workshop on Deep-Inelastic Scattering and Related Subjects (DIS2018)

16-20 April 2018

Kobe, Japan

*Speaker.

$\dagger$ On behalf of the ATLAS Collaboration, which approved this document as ATL-PHYS-PROC-2018-059. I would like to thank the analyzers who carried out the studies presented here; as well as the members of ATLAS, whose work made possible the collection of the data analyzed. 


\section{Angular analysis of $B_{d} \rightarrow K^{*} \mu^{+} \mu^{-}$decays in $p p$ collisions at $\sqrt{s}=8 \mathrm{TeV}$ with the ATLAS detector}

The $B_{d} \rightarrow K^{*} \mu^{+} \mu^{-}$decay is relevant because it involves Flavour Changing Neutral Currents (FCNC) in the $\bar{b} \rightarrow \bar{s} \mu^{+} \mu^{-}$transition. Therefore it is strongly supressed at tree level and only higher order Feynman diagrams contribute; figure 1a shows one of those processes.



(a) The diagram above shows a process allowed by the SM, that contributes to the $B_{d} \rightarrow K^{*} \mu^{+} \mu^{-}$yield.



(b) The diagram above shows the definitions of the angles in the $B_{d} \rightarrow K^{*} \mu^{+} \mu^{-}$decay.

Figure 1: Figures taken from [3].

The goal of this analysis [3] is to measure angular distributions associated to the $B_{d} \rightarrow K^{*} \mu^{+} \mu^{-}$ decay. These distributions are parametrized by $F_{L}$, the fraction of longitudinally polarized $K(892)^{*}$ mesons (from now on referred as $K^{*}$ ), and the parameters $S_{3}, S_{4}, S_{5}, \ldots, S_{9}$.

LHCb has studied this decay and found discrepancies between data and SM predictions with a 3.4 $\sigma$ significance [5]; this is another reason why this particular decay is of interest. CMS has performed this study as well [6].

\subsection{Analysis Description}

The data used to perform this measurement are $20.3 \mathrm{fb}^{-1}$ of proton-proton collisions collected by the ATLAS detector in 2012. The $B_{d} \rightarrow K^{*} \mu^{+} \mu^{-}$decays are parametrized by three angles and the mass of the system as shown in figure $1 b$.

In the diagram $\theta_{L}$ is evaluated in the center of mass of the dimuon system; $\theta_{K}$, in the center of mass of the $K^{+}$and $\pi^{-}$mesons and $\phi$ in the center of mass of the $B$ meson. The analysis is performed in different bins of the dimuon invariant mass $q^{2}$.

The angular dependence of the differential cross-section associated to the P-wave can be seen in equation 1.1, where all the angular parameters already mentioned are used.

$$
\begin{aligned}
& \frac{1}{d \Gamma / d q^{2}} \frac{d^{4} \Gamma}{d \cos \theta_{L} d \cos \theta_{K} d \phi d q^{2}}=\frac{9}{32 \pi}\left[\frac{3\left(1-F_{L}\right)}{4} \sin ^{2} \theta_{K}+F_{L} \cos ^{2} \theta_{K}+\frac{1-F_{L}}{4} \sin ^{2} \theta_{K} \cos 2 \theta_{L}\right. \\
& -F_{L} \cos ^{2} \theta_{K} \cos 2 \theta_{L}+S_{3} \sin ^{2} \theta_{K} \sin ^{2} \theta_{L} \cos 2 \phi+S_{4} \sin 2 \theta_{K} \sin 2 \theta_{L} \cos \phi+S_{5} \sin 2 \theta_{K} \sin \theta_{L} \cos \phi \\
& \left.+S_{6} \sin ^{2} \theta_{K} \cos \theta_{L}+S_{7} \sin 2 \theta_{K} \sin \theta_{L} \sin \phi+S_{8} \sin 2 \theta_{K} \sin 2 \theta_{L} \sin \phi+S_{9} \sin ^{2} \theta_{K} \sin ^{2} \theta_{L} \sin 2 \phi\right]
\end{aligned}
$$


There are large uncertainties associated to the form factors; in order to mitigate them a new parametrization $S_{i} \rightarrow P_{i}$ is chosen. However, due to low statistics, it is still not possible to use this parametrization. Additionally, a set of four trigonometric transformations, from now on called folding schemes, is used. Thus, the final set of $P_{i}^{\prime}$ parameters is obtained.

Each of the folding schemes is applied separately to the data, resulting in four datasets, any of them can be used to extract $F_{L}$ and $S_{3}$. Nevertheless, it is not possible to extract anymore $S_{6}$ and $S_{9}$. The former parameter is related to the forward-backward asymmetry through $A_{F B}=3 S_{6} / 4$.

\subsection{Event Selection}

An event needs to trigger algorithms that look for events with one, two or three muons; this requirement selects $29 \%$ of the signal events. The dimuon system is required to be consistent with having originated from a common vertex. The $K^{*}$ meson is built from two tracks with opposite charge, while the $B_{d}^{0}$ meson is formed by four tracks and is required to be displaced from the interaction vertex. Given that ATLAS cannot distinguish between kaons and pions, requirements are imposed on the $K \pi$ system's mass to tag the meson as $B_{d}^{0}$ or $\bar{B}_{d}^{0}$. When multiple candidates are found in an event, the one with the smallest $\left|m_{K \pi}-m_{K^{*}}\right| / \sigma\left(m_{K \pi}\right)$ is kept. The mistagging is also reduced by retaining the $B$ meson with the smallest $B$ vertex fit $\chi^{2}$.

The dimuon invariant mass is also used to define two control regions; where the two muons originate from the decay of a $J / \psi$, in $q^{2} \in[8,11] \mathrm{GeV}$ and where they come from the decay of a $\psi(2 S)$, in $q^{2} \in[12,15] \mathrm{GeV}$. Contributions from $\phi(1020)$ are excluded by removing any candidate with $q^{2} \in[0.98,1.1] \mathrm{GeV}^{2}$. Otherwise, the signal region encompasses $q^{2} \in\left[4 m_{\mu}^{2}, 6 \mathrm{GeV}\right]$, excluding anything above to avoid the charmonium low mass tail contamination.

\subsection{Analysis}

Once the events in data have been selected, they are fitted to PDFs representing the background and the signal components. The mass distribution of the background is modelled by an exponential PDF, while the angular dependence is modelled by second order Chebyshev polynomials in $\cos \left(\theta_{L}\right), \cos \left(\theta_{K}\right)$ and $\phi$. Any disagreements associated to the choice of PDFs is accounted for as systematics.

The signal's mass depencence is modelled with a modified Gaussian PDF with mean $m_{0}$ and width $\xi \sigma$, where $\sigma$ is the error on the mass of the $B$ candidate, while $m_{0}$ and $\xi$ are extracted from fits to the control regions mentioned above. The angular dependence is taken as the decay amplitude of the P-wave (see 1.1) after a given folding scheme is applied. The angular dependence of the acceptance is modelled also by polynomials in the angles and is extracted from simulated samples.

\subsection{Results}

The measured angular parameters are consistent with the Standard Model expectations. Figure 2 shows that $P_{4}^{\prime}$ and $P_{5}^{\prime}$ are $2.7 \sigma$ away from the prediction of Descotes-Genon et al. (DHMV) [7] in the $q^{2} \in[4,6] \mathrm{GeV}$ bin; while $P_{8}^{\prime}$ shows a small disagrement in the $q^{2} \in[2,4] \mathrm{GeV}$ bin. This is also consistent with what LHCb observed. 


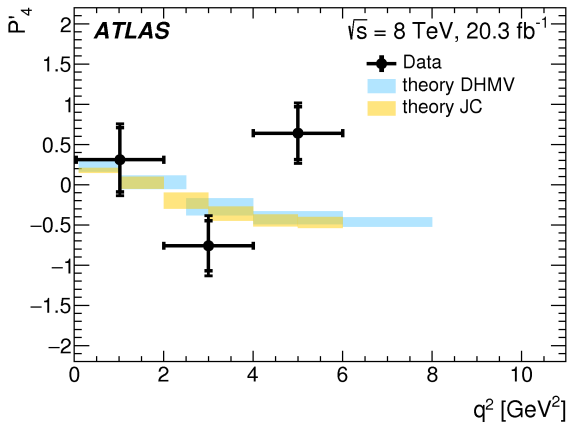

(a)

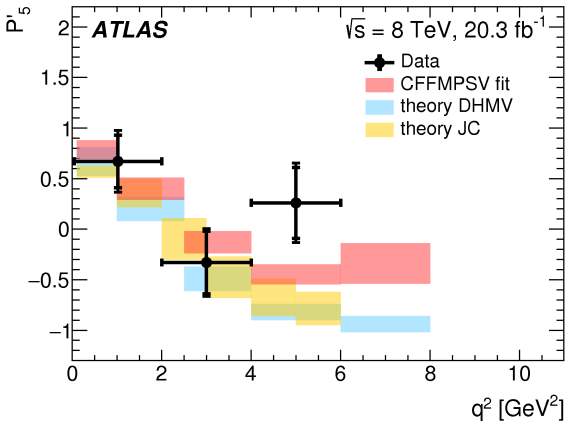

(b)



(c)

Figure 2: The three figures represent the comparison between data and different theory predictions for $P_{4}^{\prime}, P_{5}^{\prime}$ and $P_{8}^{\prime}$. Figures taken from [3].

\section{Measurement of $b$-hadron pair production with the ATLAS detector}

The goal of this analysis [4] is to measure the differential cross-section for $b \bar{b}$ production down to zero opening angle. Constraining the theoretical estimates of this cross-section is important because $g \rightarrow b \bar{b}$ is the main source of background for the $h \rightarrow b \bar{b}$ Higgs decay. Furthermore, as the LHC center of mass energy increases, the Higgs boson becomes more boosted, giving rise to smaller angles between the $b$ and $\bar{b}$ quarks.

\subsection{Analysis Description}

The strategy of this analysis is to measure the first $b$ quark through the $J / \psi \rightarrow \mu^{+} \mu^{-}$decay, where the $J / \psi$ meson originates from a $B$ meson directly or through some $c \bar{c}$ excited state. The second $b$ quark is measured through the muon in the semileptonic decay $b \rightarrow c \rightarrow \mu+X$. The cross-section is binned in several observables like $\Delta R(J / \psi, \mu), \Delta y(J / \psi, \mu), p_{T}(J / \psi, \mu)$, among others.

\subsection{Event Selection}

This analysis uses $11.4 \mathrm{fb}^{-1}$ of proton proton collisions collected by ATLAS in 2012 at $\sqrt{s}=$ $8 \mathrm{TeV}$. These events are required to trigger an algorithm designed to recognize two muons originating from a $J / \psi$ meson. The muons are built by combining Inner Detector tracks with Muon 
Spectrometer segments and are required to satisfy standard quality criteria. The reconstructed $J / \psi$ is required to be formed from two oppositely charged muons, geometrically matched to the muons built by the trigger algorithms. The third muon is taken as the one with the highest $p_{T}$ that was not used to build the $J / \psi$ meson.

\subsection{Analysis}

In order to estimate the fiducial yield of events containing a $J / \psi \rightarrow \mu^{+} \mu^{-}$decay, one has to apply corrections to compensate for trigger and muon reconstruction inefficiencies. Apart from the combinatorial background, associated to the fake $J / \psi$ background, there are two components present in data, one with $J / \psi$ mesons originating from the primary interaction, known as prompt and another, originating from the decay of $B$ mesons, known as non-prompt. In order to separate the prompt, non-prompt and fake $J / \psi$ contributions, a $2 \mathrm{D}$ fit is used, whose projections in the $J / \psi$ mass and lifetime are shown in figure 3. The second ingredient, the yield of events with
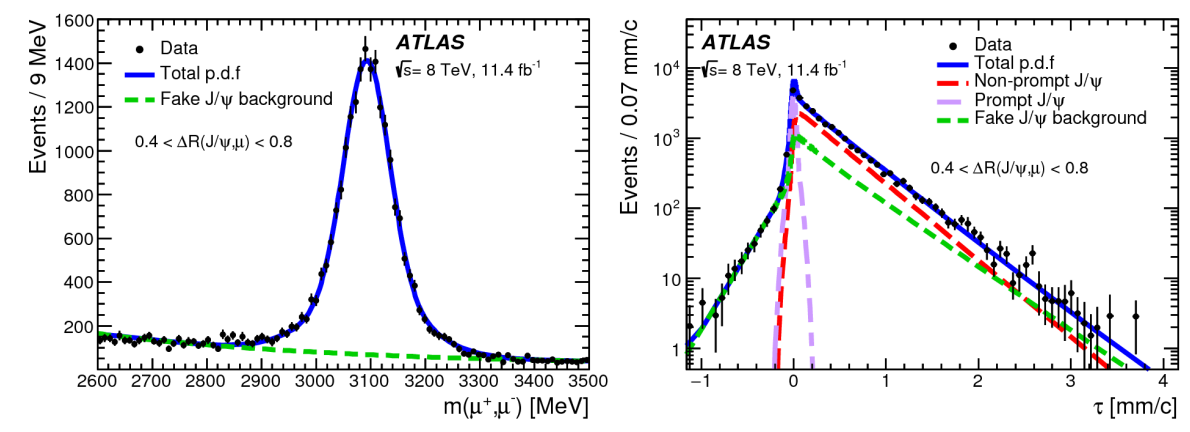

Figure 3: Projection of 2D fit to $J / \psi$ candidates in data. Figures taken from [4].

a $J / \psi \rightarrow \mu^{+} \mu^{-}$decay and a third non-prompt muon, is obtained also with a 2D fit. In order to remove the contribution of prompt $J / \psi$ decays, and thus increase the proportion of signal, a requirement of $\tau>0.25 \mathrm{~mm} / c$ is applied to the $J / \psi$. The fake $J / \psi$ yield from the previous fit is used to constrain the fake $J / \psi$ component, while the other background components are estimated from data as shown in figure 4. In this case, the discriminating variables are a BDT score, used to
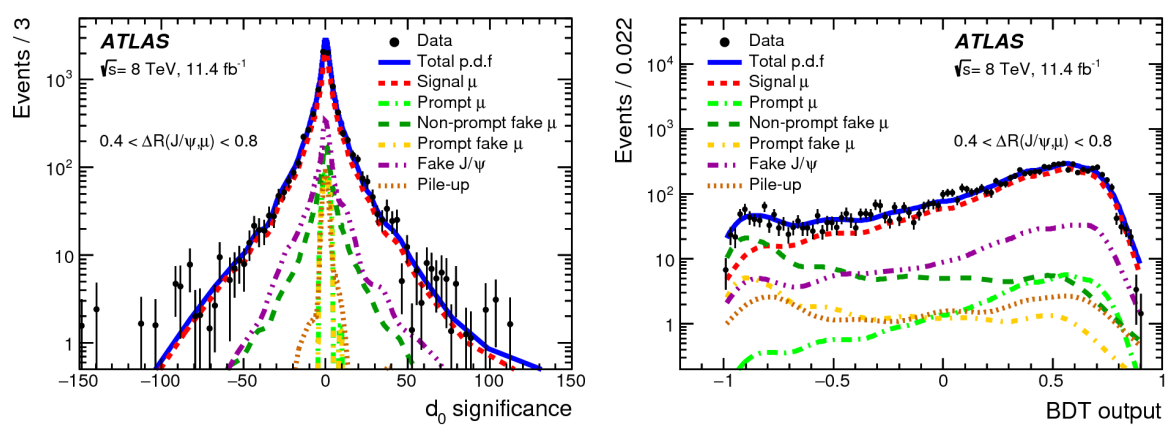

Figure 4: 2D fit to muons to data. The left plot shows the projection in the $S_{d 0}$, the right plot shows the fit in the BDT score. Figures taken from [4]. 
distinguish fake from true muons and the $d_{0}$ significance, which discriminates between prompt and non-prompt muons.

\subsection{Results}

The measured fiducial cross-section is $17.7 \pm 0.1$ (stat) \pm 2.0 (syst) nb. Due to the lack of accuracy of the leading order calculations used to produce the simulated samples, the comparison with the theoretical expectation is done using the normalized differential cross-section, instead of the differential cross-section. Figure 5 shows the predictions of several generators and data for two regimes in $p_{T}$.
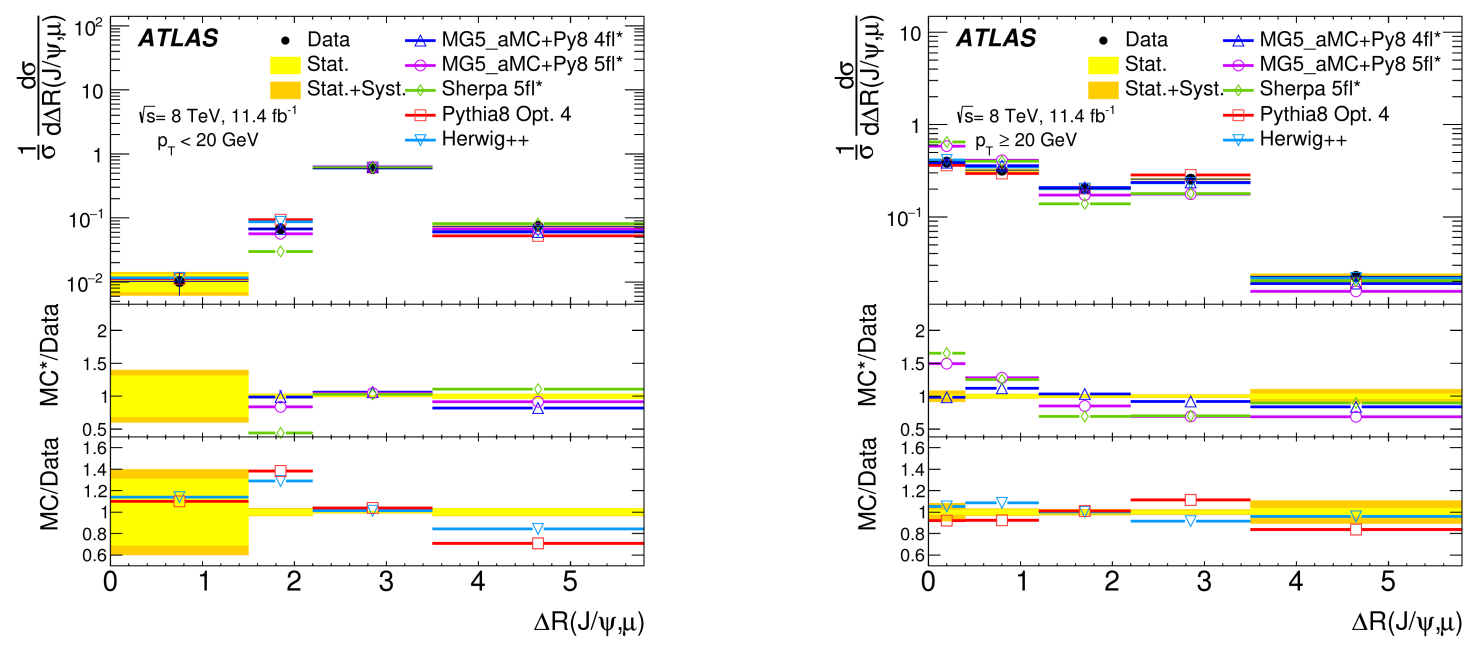

Figure 5: Comparison of differential cross-sections in data and simulation, for $p_{T}<20 \mathrm{GeV}$ (left) and $p_{T} \geq 20 \mathrm{GeV}$ (right). Figures taken from [4].

\section{References}

[1] L. Evans and P. Bryant, LHC Machine, 2008 JINST 3 S08001.

[2] ATLAS Collaboration, The ATLAS Experiment at the CERN Large Hadron Collider, 2008 JINST 3 S08003.

[3] ATLAS Collaboration, Angular analysis of $B_{d}^{0} \rightarrow K^{*} \mu^{+} \mu^{-}$decays in pp collisions at $\sqrt{s}=8 \mathrm{TeV}$ with the ATLAS detector, CERN-EP-2017-161 [hep-ex/1805.04000].

[4] ATLAS Collaboration, Measurement of b-hadron pair production with the ATLAS detector in proton-proton collisions at $\sqrt{s}=8 \mathrm{TeV}$, JHEP 11 (2017) 62 [hep-ex/1705.03374v2]

[5] LHCb Collaboration, Angular analysis of the $B^{0} \rightarrow K^{*} \mu^{+} \mu^{-}$decay using $3 \mathrm{fb}^{-1}$ of integrated luminosity, JHEP 02 (2016) 104 [hep-ex/1512.04442]

[6] CMS Collaboration, Measurement of angular parameters from the decay $B^{0} \rightarrow K^{* 0} \mu^{+} \mu^{-}$in proton-proton collisions at $\sqrt{s}=8 \mathrm{TeV}$, Phys. Lett. B 781 (2018) 517 [hep-ex/1710.02846]

[7] S. Descotes-Genon, L. Hofer, J. Matias and J. Virto, On the impact of power corrections in the prediction of $B_{d} \rightarrow K^{*} \mu^{+} \mu^{-}$observables, JHEP 12 (2014) 125. 